\title{
LAS CLASES DE LABORATORIO DE FISICA: UNA PROPUESTA PARA SU MEJORA
}

SEBASTIA, J.M.

Departamento de Física

Universidad Simón Bolívar (Venezuela)

\section{SUMMARY}

In this article we expose the features of a non-traditional design of teaching laboratory physics.

The proposed design is being applied in the teaching of bachelors in physics education at the Universidad uSimón Bolívar», Caracas. Venezuela.

\section{INTRODUCCION}

La enser̃anza de la Fisica, en tanto que ciencia experi* mental, conlleva la combinación de clases de teoria, en las que se exponen los conceptos y leyes fundamentales, y clases de laboratorio que acercan al estudiante a la experimentación y contrastación de dichas leyes.

Las clases de laboratorio resultan frecuentemente más costosas para las instituciones que las clases de teoría, tanto en recursos humanos, debido a que usualmente requieren una proporción alumno-profesor menor que la de una clase expositiva, como en recursos materiales, por la necesidad de utilizar aparatos y equipos (os* ciloscopios, carriles de aire, balanzas, etc.) para llevar a cabo los experimentos. Sin embargo, a pesar de los esfuerzos que realizan las Instituciones Educativas para una implementación adecuada de las clases de labo. ratorio, los resultados son habitualmente poco satisfactorios, tanto desde el punto de vista del estudiante, como desde la perspectiva del profesor (Shott, 1974, Buckwald, 1977, Reif \& St John, 1979).

En el presente artículo exponemos la necesidad de revisar los propósitos para los que están diseñadas las clases de laboratorio, ajustándolos a las metas principales de las carreras a las que se encuentran asociados, y presentamos una experiencia de disento no tradicional adaptado a la formación de docentes en el área de la Física que se ha implementado en la Universidad «Simón Bolívarn de Caracas, Venezuela.

\section{DIFERENTES ENFOQUES PARA EL DISEÑO DE LAS CLASES DE LABORATORIO DE FISICA}

Un punto de partida en los intentos de mejorar las clases de laboratorio reside en un aspecto clave y no siem. pre suficientemente atendido: determinar con precisión los objetivos que se desean lograr con las clases de laboratorio.

Las posibles metas que, se supone, pretenden alcanzarse en las clases de laboratorio pueden ser clasifica. das en tres grandes gnupos: a) Ilustrar el contenido de las clases teóricas, b) Enseñar técnicas experimentales y c) Promover actitudes científicas.

Prácticamente todos los diseños de clases de laboratorio pretenden alcanzar en mayor o menor grado todas las metas anteriores, sin embargo, el hecho de no explicitarlas y establecer la jerarquía de importancia que se les asigna dificulta la implementación de diseffos adecuados a la evaluación de los resultados que con ellos se logren.

Por otra lado, aún estando de acuerdo en la conveniencia de alcanzar las metas anteriores, algunos autores (Toothacker, 1983) han comprobado que otras estrategias alternativas a las clases de laboratorio permiten alcanzar dichas metas igual o más eficientemente que las clases de laboratorio habituales.

Parece conveniente, por lo tanto, prestar mayor aten- 
ción a las metas que se pretenden lograr en las clases de laboratorio, tanto para orientar el diseño instruccional de las mismas, como para establecer un patrón de referencia que permita evaluar la eficiencia global del conjunto.

Dos orientaciones básicas parecen subyacer a los diseños de clases de laboratorio de Física (Robinson, 1979). Dichas orientaciones están relacionadas, en cierta medida, con los objetivos básicos adoptados, pero no totalmente condicionados por ellos.

Una de las orientaciones más habituales es la denomidada "académica» o "tradicional», también llamada, menos formalmente, "de recetas de cocina». Dentro de esta perspectiva al estudiante se le dice exactamente lo que debe hacer, los pasos que debe seguir y los resultados que se esperan. El estudiante tiene que realizar el experimento en un tiempo fijo y no tiene oportunidad de pensar o intentar diferentes estrategias. Como ha señalado Robinson, en el laboratorio «académico» está prohibido experimentar.

La otra orientación es la denominada «experimental» también llamada "poco estructurada» o de "proyec" tom. En esta perspectiva las instrucciones se reducen a señalar las metas que se esperan y sugerir algún que otro método, el estudiante tiene tiempo flexible para pensar distintos planes de acción alternativos y llevarlos a la práctica cometiendo y corrigiendo errores. La discusión con un profesor permite orientar la actividad del estudiante e impedir planes de acción infructuosos.

\section{FUNDAMENTACION DE LA PROPUESTA Y MARCO DE LA IMPLEMENTACION}

Sin duda, la manera de concebir la ciencia y su función en la sociedad condiciona fuertemente los métodos de enseñanza de la misma.

Cuando la Ciencia es contemplada como producto de la actividad científica, que es implícitamente la posición más aceptada actualmente, el énfasis recae en los contenidos, los programas se tornan exhaustivos y los textos enciclopédicos. Por el contrario cuando la Ciencia es contemplada como proceso de comprensión e indagación de la naturaleza el acento recae en la metodología de investigación y en la resolución de problemas.

Desde esta segunda perspectiva, que nosotros compartimos, las clases teóricas, y sobre todo las experimentales, debieran estar orientadas a presentar la Física como proceso de indagación de la naturaleza, entendida esta como desarrollo de habilidades para identificar y definir un problema, formular hipótesis, disentar estrategias de resolución, recoger datos, etc., al tiempo de intentar transmitir una serie de actitudes tales como curiosidad, deseo de experimentar, acostumbrarse a du* dar de ciertas afirmaciones, etc.
Consideramos que el anterior planteamiento deberia inspirar la orientación de las clases de laboratorio de Física en todos los niveles, en particular en las carreras que forman cientificos y en especial en las carreras que forman a los docentes de materias científicas.

La función social multiplicadora que cumplen los docentes de Física parece aconsejar que sean formados dentro de un espíritu de análisis crítico y sistemático de los fenómenos naturales, actitudes que en un futuro deberán transmitir a los estudiantes, en lugar de adoctrinarlos dentro de un cuerpo rígido de leyes y teorias.

En Venezuela existe una clara diferenciación entre la carrera de Licenciatura en Física, dedicada a la formación de recursos humanos en el área de la Investigación y la docencia especializada, y la Licenciatura Docente en Física, orientada a la formación de profesores de Física, de Bachillerato, Institutos Tecnológicos y niveles básicos de las Universidades del país.

La Universidad "Simón Bolívar» de Caracas, ofrece las dos carreras antedichas haciendo uso de una orientación metodológica afín a los perfiles profesionales de los graduados que se pretende formar.

La Licenciatura en Física se imparte por la modalidad de clases expositivas o presenciales, aunque un reducido grupo puede elegir el método Keller para las materias básicas de Física, y las clases de laboratorio son, a nivel introductorio, del tipo que antes denominamos «tradicional» o «académico».

La Licenciatura Docente en Fisica, utiliza un Sistema Personalizado de Instrucción (variante del método Ke llet) para las materias de todas las áreas, Física, Matemática y Formación Docente, y ha puesto en práctica para la enseñanza de los aspectos experimentales de la Física el enfoque denominado "poco estructurado" o de «proyectos» cuyas características básicas señalábamos anteriormente.

El sistema Personalizado de Instrucción, utilizado en la Licenciatura Docente en Física, se caracteriza por la utilización, en lugar de clases expositivas, de una serie de unidades redactadas por profesores de la Universidad "Simón Bolívar» conteniendo los objetivos principales, el contenido de la unidad y algunas pruebas de autoevaluación. La Universidad pone a disposición de los estudiantes un grupo de profesores, auxiliares docentes (estudiantes graduados), y preparadores (estudiantes avanzados), que sirven de apoyo al estudiante para el aprendizaje de la unidad. Cuando el estudiante considera que tiene suficiente dominio sobre la materia presenta un examen sobre la unidad y si no alcanza los objetivos propuestos es invitado a presentar nuevamente la misma unidad sin sentirse presionado por el factor tiempo.

Dentro de este marco institucional se ha elaborado un diseño de laboratorio totalmente inspirado en la pers. 
pectiva que contempla a la Ciencia como un proceso de indagación de la naturaleza. El reducido número de alumnos y la flexibilidad de tiempo para el avance del alumno favorecieron un diseno abierto que permitiese un amplio margen al estudiante para experimentar. Los experimentos debían cumplir requisitos de ser sencillos y atractivos, no requerir equipos costosos y sofisticados y especialmente plantear a los estudiantes un cierto "problema desafío" que tuviesen que solucionar diseñando una estrategia experimental apropiada.

\section{ALGUNAS EXPERIENCIAS CONCRETAS}

Las anteriores ideas han sido plasmadas en diferentes "problemas-desafío" que han canalizado la actividad de los estudiantes en el sentido de una verdadera labor de indagación científica.

Hemos procurado que, en los experimentos propuestos, el estudiante se encuentre en una situación simiIar, en cierto modo, a la del científico que se enfrenta a una situación desconocida. Una vez que se haya definido el problema y analizadas las hipótesis, los conocimientos teóricos que posee el estudiante le permitirán elaborar una estrategia apropiada que le podrá conducir a una respuesta correcta, dentro del consiguiente margen de error, a la incógnita planteada.

A continuación presentamos un par de las experiencias diseñadas que pretenden plasmar las ideas anteriores.

\section{Experiencia $\mathrm{n}^{\circ} 1$}

\section{Tamaño de un agujero negro}

Los agujeros negros son un tema de moda en la astrofísica. Evidentemente este ejercicio no se refiere a esos agujeros negros, sino a un agujero negro mucho más modesto y sencillo.

Se le entregará un objeto de aluminio que en su interior contiene un hueco. Su tarea: sin destruir el objeto, determinar el volumen del hueco.

Usted mismo tendrá que imaginarse un procedimiento. Todo está permitido, menos tomar una sierra, cor* tar el objeto y medir el tamaño del agujero.

Mediante este ejercicio pretendemos principalmente dos cosas:

1. Que usted se ejercite en el uso de su imaginación creadora

2. Que demuestre que efectivamente sabe manejar números afectados de incertezas.

En efecto, lo que usted tiene que hacer es predecir el tamano del agujero que hay en el interior del objeto. Su predicción se basará en mediciones que usted tendrá que hacer, mediciones que, como ya sabe, estarán infectadas de incertezas.

No vacile en conversar con nosotros respecto al diseno de su experimento. No se lo disenamos nosotros mis- mos porque esta es la parte más entretenida de la Física Experimental, y porque no queremos convertirlo en un simple seguidor de instrucciones. Pero lo invitamos a conversar, porque tampoco queremos que este ejercicio le tome un tiempo demasiado largo. ¿Buena suerte!

\section{Experiencia $n^{\circ} 2$}

\section{Velocidad de una rueda de Bicicleta}

Existen muchas formas de determinar la velocidad con que gira una rueda de bicicleta. Quizás la forma más sencilla de hacerlo es a partir de la distancia que reco. rre la bicicleta y el tiempo empleado en hacerlo.

Sin embargo, en este caso no le vamos a proponer que se de un paseo en bicicleta, nuestro propósito es otro. Tendrá a su disposición una rueda de bicicleta (iuna sola!) suspendida del eje, mediante una cuerda, del techo del laboratorio.

Su tarea: determinar la velocidad angular de la rueda haciendo uso de la velocidad de precesión de la misma.

Posiblemente usted ya se habrá dado cuenta que el arre glo experimental que le of recemos no es más que el de un giróscopo de fabricación casera. Usted, que ya conoce la teoría del giróscopo, no tendrá dificultades en reiacionar la velocidad con que precesará la rueda al hacerla girar, con la velocidad de rotación de la rueda.

Mediante este ejercicio nos proponemos varias cosas:

1. Que usted adquiera información de primera mano de los misterios del movimiento giroscópico.

2. Que revise las relaciones entre las diferentes mag. nitudes que intervienen.

3. Que demuestre que conoce todo lo relativo a la propagación de errores.

Desarrolle su imaginación para diseñar el procedimiento más adecuado para dar respuesta al desafío planteado. Si tiene dificultades, no dude en venir a conversar con nosotros. ¡Buena suerte!

\section{CONCLUSIONES}

Hemos querido mostrar, con las dos experiencias an. teriores, cómo es posible diseñar experiencias de laboratorio de muy bajo costo, pero que ilustran leyes importantes de la Física y, sobre todo, aproximan al es. tudiante a adoptar una actitud similar a la del científico enfrentado a un problema: formular hipótesis, recurrir a teorías, diseffar estrategias y procedimientos experimentales, etc.

Aunque el número de estudiantes que ha estado involucrado en este tipo de experiencias es reducido, y por lo tanto insuficiente para poder argumentar a su favor aduciendo datos estadísticos, tanto estudiantes como profesores se han sentido notablemente más satisfechos participando en estos laboratorios que en las clases tradicionales de laboratorio. 
Una limitación importante de este diseño de laboratorio es que resulta difícil de extender a grupos grandes de alumnos, ya que está enfocado principalmente a ser un sistema de laboratorio personalizado.

Esperamos que experiencias similares aporten nuevas ideas para una continua mejora de la enseñanza en los laboratorios de Física.

\section{Reconocimientos}

Deseo dar las gracias al profesor Dario Moreno que ha sido el principal promotor de esta tendencia para el diseño de las clases de Laboratorio de Física en la Universidad "Simón Bolívar». También deseo agradecer la colaboración del profesor José Antonio Espinosa que participó en la elaboración de algunas de las experiencias.

\section{REFERENCIAS BIBLIOGRAFICAS}

BUCKWALD, R. A., 1977, Physics teaching as a laboratory experiment, American Journal of Physics Vol. 45, pp. 893-895.

REIF, F. E. y STJOHN, M., 1979, Teaching Physicist's thinking skills in the laboratory, American Journal of Physics. Vol. 47, pp. 950-957.

ROBISON, M.C. 1979, Undergraduate laboratories in physics: two philosophies, American Journal of Physics. Vol 47, pp. 859-862.

SHOTT, M., 1974, Physics in the Open University. Contemporary Physics, Vol. 14, pp. 69-85.

TOOTHACKER, W.S., 1983, A critical look at introductory laboratory instrution, American Journal of Physics. Vol. 51, pp. 516-520. 